\title{
Análise de custo-efetividade do ácido zoledrônico na prevenção da fratura osteoporótica proximal de fêmur no cenário do Sistema Suplementar de Saúde Brasileiro
}

\author{
Cost-effectiveness analysis of zoledronic acid for the prevention of \\ osteoporotic hip fracture in the private health care system in Brazil

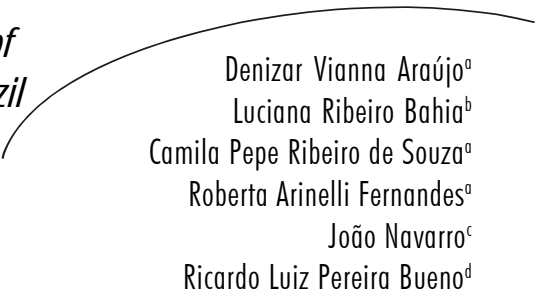

\section{Resumo}

Objetivo: realizar análise de custo-efetividade do ácido zoledrônico na prevenção de fraturas osteoporóticas proximais de fêmur. Método: modelo analítico de decisão para comparar ácido zoledrônico versus risedronato, na prevenção de fraturas, no ano 2007. A população-alvo da análise foi uma coorte hipotética de mulheres com osteoporose, idade inicial de 65 anos, horizonte de tempo de cinco anos. Dados epidemiológicos e eficácia dos fármacos foram obtidos de revisão e análise crítica da literatura. Custos foram valorados para o Sistema Suplementar de Saúde. O desfecho analisado foi o custo por fratura de fêmur evitada. Resultados: no cenário base, o ácido zoledrônico reduziu a incidência de fraturas (0,297 fraturas versus 0,460 fraturas), com custo anual do ácido zoledrônico de $\mathrm{R} \$ 21.630,85$ versus $\mathrm{R} \$$ 21.872,55 do risedronato. Conclusão: o uso do ácido zoledrônico comparado ao risedronato pode prevenir mais fraturas proximais de fêmur, com menores custos, no cenário do Sistema Suplementar de Saúde.

\section{Abstract}

Objective: To assess cost-effectiveness of zoledronic acid for the prevention of osteoporotic hip fracture. Methods: Decision analytic model to compare zoledronic acid versus risedronate, for the prevention of fracture, in 2007. The target population was a hypothetic cohort of women with osteoporosis aged 65 years in a time horizon of five years. The epidemiological data related to osteoporosis and drug's efficacy were obtained from critical appraisal of scientific literature.
Palavra-chave:

Prevenção Secundária. Fraturas do

Fêmur.

Osteoporose.

Mulheres. Avaliação

de eficácia-

efetividade de

intervenções.

Ácidos. Ações

Farmacológicas.

Análise custo-

benefício. Estudo

Comparativo.

Estudos

Epidemiológicos.

Sistemas de Saúde

\footnotetext{
Universidade do Estado do Rio de Janeiro

Departamento de Clínica Médica

Rio de Janeiro, RJ, Brasil

Correspondência / Correspondence

Denizar Vianna Araújo

E-mail: denizarvianna@medinsight.com
} 
The costs were collected from electronic claims databases of patients enrolled in Brazilian health plans. The outcome analyzed was the cost per hip fracture avoided. Results: In the base case scenario, zoledronic acid reduced the incidence of fractures ( 0.297 fractures against 0.460 fractures), with annual costs of $\mathrm{R} \$$ $21,630.85$ versus $\mathrm{R} \$ 21,872.55$ for the risedronate. Conclusions: The use of zoledronic acid compared to risedronate could prevent more hip fractures, with less costs in the Brazilian private health system.
Key words: Secondary Prevention. Femoral Fractures.

Osteoporosis. Women. Evaluation of the efficacy-effectiveness of interventions. Acids. Pharmacologic actions. Cost-benefit analysis. Comparative Study. Epidemiologic Studies. Health Systems

\section{INTRODUÇÃO}

A osteoporose é uma doença osteometabólica caracterizada pela diminuição da densidade mineral óssea e alterações na sua microestrutura que conduzem à perda da qualidade e conseqüente aumento da fragilidade óssea e tendência de fraturas por traumas de baixo impacto.

Não existem dados gerais de prevalência da osteoporose no Brasil. Alguns estudos locais de prevalência da osteoporose foram realizados nas cidades de Campinas (nos anos 2000 e 2001), São Paulo (ano 2000), São Leopoldo, no Rio Grande do Sul (nos anos 1999 e 2000) e na Ilha de Paquetá, no Rio de Janeiro (ano 2003). Costa-Paiva et al. ${ }^{1}$ analisaram 473 mulheres pós-menopausa e demonstraram prevalência de osteoporose na coluna e no fêmur de $14,7 \%$ e 3,8\%, respectivamente. Camargo $e \mathrm{al}^{2}$ realizaram estudo transversal em 301 indivíduos $\geq 70$ anos (207 mulheres e 94 homens) na cidade de São Paulo e identificaram prevalência de osteoporose em quadril e coluna lombar, respectivamente, de 6,4 e $16,1 \%$ entre os homens e 22,2 e $33,2 \%$ entre as mulheres. Zanette et al. ${ }^{3}$ avaliaram 810 laudos de densitometria óssea no sítio femoral e encontraram uma prevalência de 4,0\% em mulheres abaixo de 50 anos e 13,9\% em mulheres com idade igual ou acima de 50 anos com resultados de $t$-score abaixo de $\leq-2,5$ desvios-padrão. Oliveira et al. ${ }^{4}$ utilizaram a técnica de ultra-sonometria óssea de calcâneo para avaliar 385 mulheres na pós-menopausa e demonstraram que $16,8 \%$ da população apresentava T-score $\leq-2,5$, com aumento progressivo com a idade. Nesses estudos, os métodos utilizados para o diagnóstico da osteoporose, as populações estudadas, assim como os desenhos dos estudos, foram diferentes, não permitindo generalização dos resultados para toda população brasileira.

A complicação mais temida da osteoporose é a fratura proximal de fêmur. Esta fratura resulta em mortalidade $15 \%$ maior no primeiro ano pós-fratura, comparativamente ao grupo não afetado de idade similar. ${ }^{5} \mathrm{Os}$ pacientes com fratura prévia são duas a cinco vezes mais susceptíveis a fraturas futuras do 
que indivíduos sem fraturas. ${ }^{6} \mathrm{~A}$ incidência anual de fratura proximal de fêmur em mulheres acima de 60 anos na cidade de Marília, São Paulo, em 1995, foi de 50,03/10 mil habitantes: ${ }^{7}$ na cidade de Sobral, Ceará, nos anos de 1996/2000, foi de 12,4/10 mil habitantes; ${ }^{8} \mathrm{e}$ na cidade de Fortaleza, Ceará, no ano 2001/ 2002 foi de 21,7/10 mil habitantes. ${ }^{9}$

A fratura proximal de fêmur é causa de importante ônus econômico para os sistemas de saúde. O Sistema Suplementar de Saúde representa o setor privado de assistência à saúde no Brasil no qual operadoras de planos de saúde contratam prestadores de serviços de saúde (hospitais, clínicas, consultórios, laboratórios) para atendimento de seus beneficiários. O sistema é regulado pela Agência Nacional de Saúde Suplementar e regido pela Lei Federal $n^{\circ} 9.656 / 98 .{ }^{10}$

Araújo et al. ${ }^{11}$ realizaram estudo transversal sobre a estimativa de custos para o tratamento cirúrgico da fratura osteoporótica do fêmur proximal e as complicações ocorridas durante a internação, em pacientes de ambos os sexos acima de 50 anos atendidos no Sistema Suplementar de Saúde Brasileiro, no período de julho de 2003 a junho de 2004. O custo médio total da hospitalização foi de $\mathrm{R} \$ 24$ mil, sendo o maior componente de custo atribuído ao material médico.

O uso de agentes anti-reabsortivos, principalmente os bisfosfonatos, constitui a base do tratamento farmacológico da osteoporose, sendo que apenas o alendronato, o risedronato e o ácido zoledrônico demonstraram ser capazes de reduzir o risco de fraturas não-verte- brais em estudos clínicos randomizados. Embora a prescrição de bisfosfonatos orais venha aumentando mundialmente, pela inequívoca eficácia descrita sobre os desfechos da osteoporose, a aderência ao tratamento é problemática. A necessidade de cuidados especiais para o uso e os eventos adversos gastrointestinais diminuem a aderência no longo prazo.

O ácido zoledrônico, medicação parenteral com dose única anual, torna-se uma opção terapêutica para melhorar a aderência de pacientes intolerantes aos bisfosfonatos. $\mathrm{O}$ ácido zoledrônico foi capaz de diminuir a reabsorção óssea e melhorar a densidade mineral óssea por pelo menos 12 meses após uma única infusão, sugerindo um efeito duradouro. ${ }^{12} \mathrm{O}$ estudo HORIZON avaliou os efeitos da infusão de ácido zoledrônico $5 \mathrm{mg} / \mathrm{ano}$ por três anos consecutivos na incidência de fraturas de vértebras, proximais de fêmur e outras fraturas em mulheres na pós-menopausa. ${ }^{13} \mathrm{O}$ estudo foi duplo-cego, multicêntrico, randomizado e controlado por placebo. As pacientes eram alocadas em dois grupos para receber a infusão de $5 \mathrm{mg}$ ácido zoledrônico ou placebo durante 15 minutos no período basal, após 12 e 24 meses, além de terapia adjuvante com cálcio oral $(1.000$ a $1.500 \mathrm{mg})$ e vitamina D (400 a 1.200UI).

O grupo estudado foi de 7.765 mulheres na pós-menopausa com idade média de 73 anos com $T$ score $\leq-2,5$ no colo fêmur com ou sem evidência de fratura vertebral ou $T$ score $\leq-1,5$ com evidência radiológica de fratura vertebral, sendo que 3.889 receberam ácido zoledrônico e 3.876 receberam placebo ( $81 \%$ da população recebeu as 3 infusões). 
A incidência de fratura vertebral morfométrica após três anos foi de 10,9\% no grupo placebo e 3,3\% no grupo tratado com ácido zoledrônico, ou seja, uma diminuição no risco relativo de 70\% [Redução do Risco Absoluto (RRA) $=7,6 \%$, Número Necessário para Tratar $(\mathrm{NNT})=13$ ]. A incidência de fratura proximal de fêmur foi de 2,5\% no grupo placebo e 1,4\% no grupo tratado com ácido zoledrônico, ou seja, redução do risco relativo de $44 \%(\mathrm{RRA}=1,1 \%, \mathrm{NNT}=90)$. Na análise de subgrupos de pacientes mais graves ( $T$ score $\leq-2,5)$ e menos graves (T score $>-2,5)$, observou-se redução na incidência cumulativa de fraturas proximais de fêmur em $34 \% \mathrm{e}$ $80 \%$, respectivamente. ${ }^{14}$

O objetivo deste estudo foi elaborar análise de custo-efetividade do ácido zoledrônico comparado ao risedronato, na prevenção de fraturas osteoporóticas de fêmur, no cenário do Sistema Suplementar de Saúde.

\section{MÉTODO}

Foi elaborado modelo analítico de decisão para estimar a razão de custo-efetividade do ácido zoledrônico comparado ao risedronato, no tratamento da osteoporose, no Brasil. A população-alvo da análise foi uma coorte hipotética de mulheres, idade inicial de 65 anos, com osteoporose (T score $\leq-2,5$ ou $T$ score $\leq-1,5$ com evidência radiológica de fratura vertebral), no cenário do Sistema Suplementar de Saúde. Os dados epidemiológicos da osteoporose,${ }^{1-7} \mathrm{o}$ dado de freqüência de fratura proximal de fêmur na popula- ção do Sistema Suplementar de Saúde ${ }^{11}$ e de eficácia ${ }^{12,15,16}$ dos agentes terapêuticos utilizados foram obtidos de revisão e análise crítica da literatura.

A valoração dos honorários e procedimentos médicos no Sistema Suplementar de Saúde foi feita pela Classificação Brasileira Hierarquizada de Procedimentos Médicos (CBHPM), $4^{a}$ edição, versão $2005 .{ }^{17} \mathrm{~A}$ valoração da diária hospitalar no Sistema Suplementar de Saúde foi obtida em publicação do PROAHSA (USP/GV). ${ }^{18}$ Os fármacos utilizados foram valorados pela Revista Kairos. ${ }^{19}$ Os exames complementares foram valorados segundo a CBHPM 4 edição, versão 2005. As estatísticas populacionais brasileiras foram obtidas do IBGE. ${ }^{20}$

O modelo estimou a razão de custo-efetividade incremental do ácido zoledrônico comparado ao risedronato, no cenário brasileiro, para o seguinte desfecho: custo por fratura proximal de fêmur evitada. A taxa de desconto de 3\% foi aplicada tanto para os custos futuros como para benefícios clínicos associados ao tratamento.

\section{Estrutura do modelo}

Para a estimativa dos custos e conseqüências dos tratamentos, foi elaborado um modelo que simulou a redução do risco de uma fratura proximal de fêmur e projetou o número de eventos (fraturas proximais de fêmur) esperados através de alguns estadios evolutivos, baseados em estados de Markov. Este tipo de modelo é indicado para repre- 
sentar processos aleatórios que evoluem no tempo, e é particularmente útil para se modelar o impacto da redução do risco de fratura proximal de fêmur em mulheres pós-menopausa no Brasil. Modelos desse tipo têm dois componentes: estrutura e parâmetros. A "estrutura" refere-se aos estados de saúde representados no modelo e as transições possíveis entre eles. Os "parâmetros" do modelo incluem os valores de probabilidades atribuídos às transições entre estados de saúde.

Neste estudo, as pacientes iniciam no modelo em um único estado: mulheres pós-menopausa, com 65 anos, $T$ score $\leq-2,5$ ou $\leq-1,5$ com evidência radiológica de fratura vertebral. O modelo de Markov assume que as transições entre os estados ocorrem num intervalo de um ano. Deste estado, com o pas- sar de um período, as pacientes podem permanecer neste mesmo estado, evoluir para o óbito, sofrer uma primeira fratura proximal de fêmur ou descontinuar o tratamento. As pacientes que sofreram a primeira fratura proximal de fêmur, com o passar de um período podem permanecer neste estado ou sofrer uma segunda fratura proximal de fêmur. Deste último estado, com o passar de mais um período, a paciente pode permanecer neste mesmo estado ou evoluir para o óbito. As pacientes que descontinuaram o tratamento podem, com o passar de um período, evoluir para o óbito ou sofrer uma primeira fratura proximal de fêmur. Deste estado, as pacientes podem seguir a mesma seqüência de episódios descrita anteriormente. A figura 1 resume a representação esquemática dos estados do modelo de Markov.

Figura 1 - Estados de saúde assumidos no modelo econômico de custo-efetividade

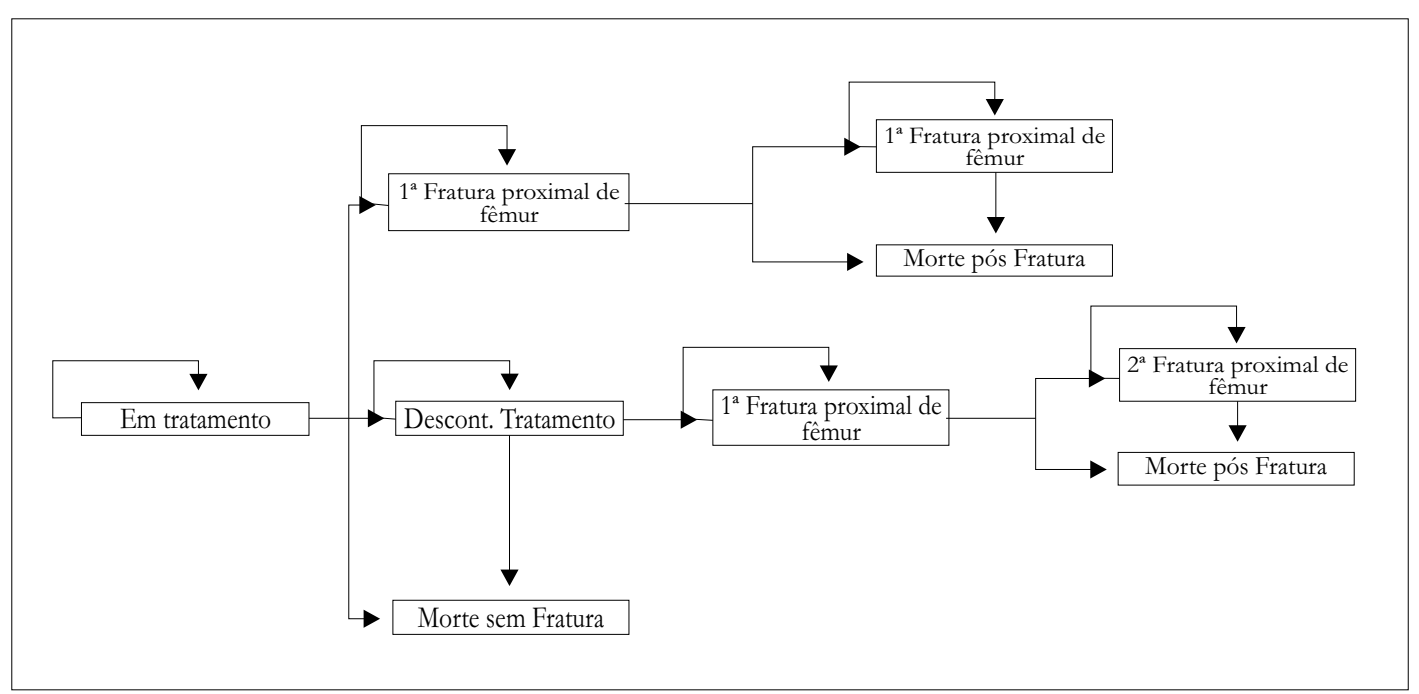


O modelo acompanhou a paciente dos 65 anos de idade no horizonte de tempo de cinco anos, considerando a tábua de expectativa de vida, por idade e sexo, disponibilizada pelo IBGE..$^{20} \mathrm{~A}$ cada ano as pacientes foram realocadas entre os estados de Markov de acordo com as probabilidades de transição entre os estados.

\section{Probabilidades}

O ácido zoledrônico deve ser administrado uma única vez ao ano, enquanto o risedronato necessita de administração semanal. Assumiu-se que a taxa de aderência das pacientes tratadas com ácido zoledrônico é de $100 \%$. A maior taxa de aderência ao tratamento encontrada na literatura para a posologia diária foi de $57 \%$, e para a semanal foi de $69,7 \%{ }^{16}$ Em estudo recente, Weycker et al. ${ }^{21}$ encontraram uma taxa de falha na adesão ao tratamento maior que $70 \%$. As estimativas para as eficácias das drogas utilizadas no modelo foram extraídas de revisão e análise crítica da literatura com níveis $1 \mathrm{~A}$ e $1 \mathrm{~B}$ da hierarquia da evidência (tabela 1).

Tabela 1 - Eficácia dos fármacos na redução de fraturas proximais de fêmur. Rio de Janeiro, RJ, 2007.

\begin{tabular}{lcc}
\hline Fármacos & Eficácia & Horizonte do estudo (anos) \\
\hline Ácido zoledrônico (12) & $41 \%$ & 3 \\
Risedronato (a) & $36 \%$ & 3 \\
\hline
\end{tabular}

A estimativa de que $17 \%$ das pacientes com fraturas em nosso meio sofrem uma fratura contralateral em um período de um ano também foi adotada pelo modelo. ${ }^{22} \mathrm{~A}$ freqüência de fraturas proximais de fêmur entre uma população de mulheres pós-menopausa no Brasil foi extrapolada do estudo nacional realizado por Araújo et al. ${ }^{11} \mathrm{~A}$ incidência de fratura proximal de fêmur em mulheres, por faixa etária ( 50 a 59 anos, 60 a 69 anos e 70 anos ou mais), foi extrapolada assumindo uma relação exponencial entre a idade e a incidência de fratura, para estimar o risco de fratura para cada idade. Para isso, os valores revelados no estudo foram considerados como correspondentes ao risco de fratura na idade média do intervalo relacionado. Sendo assim, a taxa de $1,13 \%$ e $6,86 \%$ estimadas, respectivamente, para o primeiro e o segundo grupos etários, foram consideradas como pertencentes às idades de 55 e 65 anos. Para efeito de simplificação, a taxa de $14,32 \%$ estimada no estudo para pacientes com 70 anos ou mais, foi adotada como a taxa dos pacientes com 70 anos. Assumiu-se que acima dos 70 anos a taxa é constante.

\section{Custos}

O modelo considera apenas custos médicos diretos. Entre os custos médicos diretos adotados estão: custo anual com medicamentos, cus- 
to com internação hospitalar para tratamento da fratura e custo anual de acompanhamento dos pacientes. Os custos anuais com ácido zole- drônico e risedronato estão apresentados na tabela 2. O custo do ácido zoledrônico foi acrescido do procedimento de infusão $(\mathrm{R} \$ 72,00)$.

Tabela 2 - Custo anual com Ácido Zoledrônico e Risedronato. Rio de Janeiro, RJ, 2007.

\begin{tabular}{lrrrr}
\hline \multicolumn{1}{c}{ Medicamento } & Custo Unitário & Freqüência & Custo Anual & $\begin{array}{c}\text { Custo Anual + } \\
\text { Infusão }\end{array}$ \\
\hline Ácido Zoledrônico & $\mathrm{R} \$ 1.272,15$ & 1 & $\mathrm{R} \$ 1.272,15$ & $\mathrm{R} \$ 1.344,15$ \\
Risedronato & $\mathrm{R} \$ 30,17$ & 52 & $\mathrm{R} \$ 1.568,84$ & $\mathrm{R} \$ 1.568,84$ \\
\hline
\end{tabular}

Os custos relacionados ao tratamento da fratura e da fratura contralateral subseqüente estão apresentados na tabela 3. Estes custos foram obtidos e atualizados do trabalho ori- ginal elaborado por Araújo e colaboradores, que estimaram o custo da fratura osteoporótica de fêmur no Sistema Suplementar de Saúde.

Tabela 3 - Custo com hospitalização para tratamento cirúrgico da fratura. Rio de Janeiro, RJ, 2007.

\begin{tabular}{lc}
\hline Fratura proximal do fêmur & Custo Unitário / Fratura R\$ \\
\hline Cirurgia e honorário & $1.938,00$ \\
Atendimento em Unidades de Urgência & 42,00 \\
Diária Quarto & 214,60 \\
Diária UTI & 461,50 \\
Diária Sala Cirurgia & 197,80 \\
Materiais - Prótese & $14.749,50$ \\
Medicamentos & $1.879,33$ \\
CUSTO TOTAL & $24.051,17$ \\
\hline
\end{tabular}

Assumiu-se que o custo anual de acompanhamento para pacientes com osteoporose com e sem fraturas seria o mesmo. A conduta anual adotada foi a recomendada pelo Consenso da Sociedade Brasileira de Densitometria Clínica, e seria de um exame de densitometria óssea da coluna e fêmur, um exame de creatinina, um exame de calcemia, um exame de calciúria 24 horas, uma mensuração da fosfatase alcalina, uma radiografia da coluna vertebral e duas consultas médicas. A tabela 4 apresenta os valores do acompanhamento das pacientes. 
Tabela 4 - Custo anual de acompanhamento das pacientes. Rio de Janeiro, RJ, 2007.

\begin{tabular}{lccc}
\hline Follow- $\boldsymbol{y}$ de tratamento & Custo Unitário & Freqüência & Custo Anual \\
& $\mathrm{R} \$$ & 1 & $\mathrm{R} \$$ \\
\hline Densitometria óssea coluna e fêmur & 167,88 & 1 & 4,87 \\
Creatinina & 4,53 & 1 & 4,53 \\
Calcemia & 4,53 & 1 & 4,53 \\
Calciúria 24 Horas & 4,53 & 1 & 37,31 \\
Radiografia de coluna vertebral & 37,31 & 1 & 8,36 \\
Mensuração da fosfatase alcalina & 8,36 & 8,17 & 343,14 \\
Consulta Médica & 42,00 & & 570,27 \\
CUSTO TOTAL & & & \\
\hline
\end{tabular}

\section{Desfechos clínicos}

O modelo considerou como desfecho o número de casos de fratura proximal de fêmur evitada. A razão de custo efetividade incremental (RCEI), na comparação do tratamento com ácido zoledrônico versus risedronato, baseou-se na seguinte fórmula: RCEI = (Custo Incremental) / (Efetividade Incremental). O custo incremental corresponde ao custo médio do tratamento com ácido zoledrônico menos o custo médio do tratamento com risedronato, durante o horizonte de tempo da análise. A efetividade incremental corresponde à diferença entre o total de fraturas verificadas, durante esse período de tempo, entre as pacientes tratadas com ácido zoledrônico versus risedronato.

\section{RESULTADOS}

O resultado foi apresentado em termos de custo incremental por fratura proximal de fêmur evitada.

O quadro 1 resume o resultado da análise de custo-efetividade para comparação do tratamento com ácido zoledrônico versus risedronato, para o cenário base que considera pacientes com $T$ score $\leq-2,5$ ou $\leq-1,5$ com evidência radiológica de fratura vertebral, no horizonte de tempo de cinco anos. O ácido zoledrônico reduziu o número de fraturas $(0,297$ fraturas versus 0,460 fraturas), em comparação com o risedronato. A razão de custo-efetividade incremental do tratamento com o ácido zoledrônico versus risedronato revelou ser custo-efetiva por fratura proximal de fêmur evitada em cinco anos. 
Quadro 1 - Resultado da análise de custo-efetividade do Ácido Zoledrônico versus Risedronato. Rio de Janeiro, RJ, 2007.

\begin{tabular}{|l|c|c|c|c|}
\hline & Ácido Zoledrônico & Risedronato & Incremental & \\
\cline { 1 - 4 } Medicamento & $\mathrm{R} \$ 7.168,48$ & $\mathrm{R} \$ 928,99$ & $\mathrm{R} \$ 6.239,49$ & \\
\hline Follow-up & $\mathrm{R} \$ 3.041,33$ & $\mathrm{R} \$ 3.006,21$ & $\mathrm{R} \$ 35,12$ & \\
\hline Fratura & $\mathrm{R} \$ 11.421,04$ & $\mathrm{R} \$ 17.937,35$ & $(\mathrm{R} \$ 6.516,31)$ & \\
\hline Total & $\mathrm{R} \$ 21.630,85$ & $\mathrm{R} \$ 21.872,55$ & $\mathrm{R} \$ 241,70$ & \\
\hline & Ácido Zoledrônico & Risedronato & Incremental & \multirow{2}{*}{ RCEI* $^{*}$} \\
\hline $1^{2}$ Fratura & 0,243 & 0,353 & $(0,110)$ & - \\
\hline $2^{2}$ Fratura & 0,054 & 0,107 & $(0,053)$ & - \\
\hline Fratura & 0,297 & 0,460 & $(0,163)$ & Dominante \\
\hline
\end{tabular}

*RCEI: Razão de Custo-Efetividade Incremental (Custo por fratura proximal de fêmur evitada em 5 anos)

\section{DISCUSSÃO}

No Brasil, o aumento da expectativa de vida nas últimas décadas resultou em incremento na prevalência de doenças crônicodegenerativas, entre as quais a osteoporose. O número de estudos sobre prevalência é reduzido, embora seja a doença osteometabólica mais comum. As taxas de ocorrência de osteoporose apresentaram grande amplitude, devido às características das populações estudadas em relação a etnia, sexo, idade e o sítio pesquisado - coluna lombar ou fêmur. Com isso, não existem dados sobre o real impacto dessa doença para os sistemas de saúde.

Os estudos de custo-efetividade visam a comparar diferentes intervenções em relação a suas eficácias e custos, gerando informações úteis para médicos e gestores de saúde na tomada de decisão e alocação de recursos es- cassos em um ambiente de crescentes custos com a assistência de saúde.

Neste estudo, foram comparadas duas intervenções terapêuticas (ácido zoledrônico e risedronato), reconhecidamente eficazes na redução de fraturas proximais de fêmur, em relação a seus custos no horizonte de tempo de cinco anos em uma coorte hipotética de mulheres pós-menopausa.

Foram considerados apenas os custos diretos dos desfechos, isto é, fraturas de fêmur proximal. Os custos indiretos (perda de produtividade, dependência de terceiros para atividades de rotina, atendimento domiciliar, etc) não foram contemplados no modelo, devido à inexistência de dados no nosso país, mas sem dúvida constituem um importante aspecto a ser considerado em estudos futuros. Evidências na literatura revelam que pacientes submetidos a tratamento cirúrgico da fratura 
de fêmur proximal necessitam de cuidados após a alta hospitalar, como analgesia, profilaxia do tromboembolismo, ${ }^{24}$ consultas de acompanhamento com ortopedistas e fisioterapeutas ${ }^{25,26}$ e controle radiográfico, além dos relacionados à perda da independência pósfratura. ${ }^{27,28}$ Estes custos, que se somam ao custo anual para pacientes com osteoporose, não foram considerados no modelo econômico, tornando o resultado conservador para a estratégia com ácido zoledrônico.

As diferenças na eficácia e aderência ao tratamento, obtidos de estudos clínicos randomizados e metanálises, resultaram em diminuição do número de fraturas proximais de fêmur evitadas com o ácido zoledrônico (ácido zoledrônico 0,297 vs 0,460 risedronato). $\mathrm{Na}$ análise de custo-efetividade do ácido zoledrônico versus risedronato, observou-se que o primeiro é uma opção de minimização de custos.

A aderência ao tratamento com bifosfonatos orais é um limitante no longo prazo, com taxas de adesão variando de $17.9 \%$ a $78.0 \%{ }^{16}$ A relação entre a aderência ao tratamento da osteoporose e o risco de fraturas foi avaliada em 11.252 mulheres canadenses entre 1996 e $2001 .^{29}$ Menos da metade das mulheres, para as quais as medicações eram ofertadas pelo governo, apresentavam adequada aderência ao tratamento (>80\%), e $40 \%$ pararam de usar a medica- ção oral após cinco anos de acompanhamento. As mulheres que apresentaram menor aderência ao tratamento estavam em uso de etidronato e alendronato.

Os autores concluíram que uma alta aderência ao tratamento $(>90 \%)$ é necessária para minimizar o risco de fraturas no mundo real. A preferência pelo uso de medicação uma vez ao ano deve ser considerada, assim como a possibilidade de melhor relação aderência-efetividade. Os resultados da análise econômica demonstram que o ácido zoledrônico, sendo uma opção com maior aderência, deve ser considerado opção terapêutica para minimizar o impacto econômico das fraturas osteoporóticas do fêmur proximal.

\section{AGRADECIMENTOS}

Agradecemos o auxílio financeiro da Novartis BioCiências S/A para realização deste estudo.

\section{NOTAS}

\footnotetext{
Departamento de Clínica Médica da Universidade do Estado do Rio de Janeiro e MedInsight - Rio de Janeiro, RJ, Brasil

b MedInsight - Rio de Janeiro, RJ, Brasil

c Novartis BioCiências S/A - São Paulo, SP, Brasil

d Universidade Federal do Rio Grande do Sul e Novartis BioCiências S/A - Porto Alegre, RS, Brasil
} 


\section{REFERÊNCIAS}

1. Costa-Paiva L, Horovitz AP, Santos AO, Fonsechi-Carvasan GA, Pinto-Neto AM. Prevalência de osteoporose em mulheres na pós-menopausa e associação com fatores clínicos e reprodutivos. Revista brasileira de ginecologia e obstetricia 2003; 25(7):507-12.

2. Camargo MB, Cendoroglo MS, Ramos LR, de Oliveira Latorre Mondo R, Saraiva GL, Lage A, Carvalhaes Neto N, Araujo LM. Bone mineral density and osteoporosis among a predominantly Caucasian elderly population in the city of São Paulo, Brazil. Osteoporos Int 2005;16 (11):1451-60.

3. Zanette E, Stringari FF, Machado F, Marroni BJ, Ng DPK, Canani LH. Avaliação do Diagnóstico Densitométrico de Osteoporose/Osteopenia conforme sítio ósseo. Arq Bras Endocrinol Metabol 2003;47(1):30-6.

4. Oliveira PP, Klumb EM, Marinheiro LP. Prevalência do risco de fraturas estimado pela ultra-sonometria óssea de calcâneo em uma população de mulheres brasileiras na pósmenopausa. Cad Saude Publica 2007; 23(2):381-90.

5. Parker MJ, Palmer CR. Prediction of rehabilitation after hip fracture. Age Ageing 1995;24: 96-8.

6. Solomon DH, Finkelstein JS, Katz JN, Mogun H, Avorn J. Underuse of Osteoporosis Medications in Elderly Patients with Fractures. Am J Med 2003;115:398-400.

7. Komatsu RS, Ramos LR, Szejnfeld VL. Incidence of proximal femur fractures in Marilia, Brazil. J Nutr Health Aging 2004;8(5):362-7.

8. Castro da Rocha FA, Ribeiro AR. Low incidence of hip fractures in an equatorial area. Osteoporos Int 2003 July;14(6):496-9.
9. Silveira VA, Medeiros MM, Coelho-Fiho JM, Mota RS, Noleto JC, Costa FS, Pontes FJ, Sobral JB, Aguiar RF, Leal AC, Clemente CM. Hip fracture incidence in an urban area in Northeast Brazil. Cad Saude Publica 2005; 21(3): 907-12.

10. Agência Nacional de Saúde Suplementar (Brasil). Lei de Regulamentação do Sistema Suplementar de Saúde. Lei no 9.656, de 03 de junho de 1998. [acesso em: 22 ago. 2008] Disponível em: URL: http:// www.ans.gov.br/portal/site/legislacao/ legislacao_integra.asp?id_original $=455$

11. Araújo DV, Oliveira JH, Bracco OL. Custo da Fratura Osteoporótica de Fêmur no Sistema Suplementar de Saúde. Arq Bras Endocrinol Metabol 2005;49(6):897-901.

12. Reid IR, Brown JP, Burckhardt P, Horowitz Z, Richardson P, Trechsel U, et al. Intravenous zoledronic acid in postmenopausal women with low bone mineral density. N Eng J Med 2002, 346(9):653-61.

13. HORIZON Study. Data on file - Novartis.

14. Black DM, Delmas PD, Eastell R, Reid IR, Boonen S, Cauley JA, et al. Once-yearly zoledronic acid for treatment of postmenopausal osteoporosis. N Eng J Med 2007; 356 (18):1809-22.

15. Papapoulos SE, Quandt SA, Liberman UA, Hochberg MC, Thompson DE. Metaanalysis of efficacy of alendronate for the prevention of hip fractures in postmenopausal women. Osteoporos Int 2005;16:468-74.

16. Cramer JA, Gold DT, Silverman SL, Lewiecki EM. A systematic review of persistence and compliance with bisphosphonates for osteoporosis. Osteoporos Int 2007; 18:1023-31. 
17. Associação Médica Brasileira. Classificação Brasileira Hierarquizada de Procedimentos Médicos (CBHPM). 4. ed. 2005. [acesso 2007 dez 17] Disponível em: URL: http:/ / www.amb.org.br/cbhpm_4edicao.php3

18. Universidade de São Paulo. Faculdade de Medicina. Hospital das Clínicas. Boletim de Indicadores do PROAHSA (USP/GV), 2006. [acesso 2007 dez 17] Disponível em: URL: http://www.hcnet.usp.br/proahsa/ indicadores/

19. Revista Kairos. [acesso 2007 dez 17$]$ Disponível em: URL: http:// www.revistakairos.com/revista/bra/ default_bra.asp

20. IBGE - Instituto Brasileiro de Geografia e Estatística. [acesso 2007 dez 17] Disponível em: URL: www.ibge.gov.br

21. Weycker D, Macarios D, Edelsberg J, Oster G. Compliance with osteoporosis drug therapy and risk of fracture. Osteoporos Int 2007;18(3):271-7

22. Wannmacher L. A eficácia de Cálcio e Vitamina D na prevenção de fraturas ósseas. Uso Racional de Medicamentos: Temas Selecionados 2005; 2:1-6.

23. Consenso Brasileiro em Densitometria, Sociedade Brasileira de Densitometria Clínica -SBDens Setembro de 2003. [acesso 2007 set 6] Disponível em: URL:

www.sbdens.org.br

24. Geerts WH, Pineo GF, Heit JA, Berggvist D, Lassen MR, Colwell CW, et al. Prevention of venous thromboembolism: the Seventh ACCP Conference on Antithrombotic and Thrombolytic Therapy. Chest 2004;126 (3 Suppl):338S-400S

25. Host HH, Sinacore DR, Bohnert KL, StegerMay K, Brawn M, Binder EF. Training-induced strength and functional adaptation after hip fracture. Phys Ther 2007;87(3):292-303

26. Binder EF, Brown M, Sinacore DR, StegerMay K, Yarasheski KE, Schechtman KB. Effects of extended outpatient rehabilitation after hip fracture: a randomized controlled trial. J Am Med Assoc 2004; 292 (7):837-46.

27. Pasco JA, Sanders KM, Hoekstra FM, Henry MJ, Nicholson GC, Kotowicz MA. The human cost of fracture. Osteoporos Int 2005 16: 2046-52.

28. Cunha U, Veado MAC. Fratura da extremidade proximal do fêmur em idosos; independência funcional e mortalidade em um ano. Revista brasileira de ortopedia 2006; 41 (6):195-9.

29. Caro JJ, Ishak KJ, Huybrechts KF, Raggio G, Naujoks C. The impact of compliance with osteoporosis therapy on fracture rates in actual practice. Osteoporos Int 2004;15:1003-8. 